Bangladesh J. Sci. Ind. Res. 43(1), 47-54, 2008

\title{
Influence of Nitrogen - Phosphorus Fertilization and Time of Harvest on the Growth, Yield and Oil Content of Mentha spicata $\mathbf{L}$.
}

\author{
A.A. Jahangir, K. Nada, F.Begum, M.Hossain, M.A.M. Sarker and M. Moniruzzaman \\ BCSIR Laboratories,Dhaka.Dhanmondi, Dhaka - 1205, Bangladesh
}

\begin{abstract}
Abstrat
Field experiment was conducted to evaluate the influence of application of nitrogenphosphorus fertilizer and harvesting time on growth, yield, oil content and physiochemical properties of M. spicata L. oil. Eight treatment consists of different N-P fertilizer dose and four harvesting period were used for the study. Maximum plant height was found at $\mathrm{N}_{2} \mathrm{P}_{2}$ treatment. $\mathrm{N}_{2} \mathrm{P}_{3}$ fertilizer treatment produced highest herb yield. The oil content of $M$. spicata L. was maximum at 130 day harvesting period. The oil refractive index was also enhanced with increasing harvesting time. In case of interaction effect between different level of nitrogen-phosphorus and harvesting time showed highest oil concentration at $\mathrm{N}_{2} \mathrm{P}_{2}$ treatment within 130 days harvesting time. Where as the optical rotation, acid value and density of oil was remain more or less same during the whole experiment period.
\end{abstract}

Key words : Mentha spicata, Nitrogen-phosphorus, Fertilizer, Harvest time, Optical rotation, Acid value and Oil content.

\section{Introduction}

Mentha is a small genus of aromatic herb belongs to Labiateae family. M. spicata $\mathrm{L}$. is one of the most important species of the genus mentha. It is popularly known as spearmint. The essential oil obtained from fresh shoot of $M$. spicata L. is rich in menthol, carvon, linalool and linanyl acetate, carvon being the most important constituent among all for industrial application (Sing hand Misra 2000). It is commercially exploited in perfumery, food, cosmetic flavors and pharmaceutical industries (Chattpadhyay et al 2002). The herb yields an essen- tial oil biosynthesis in $M$. spicata $\mathrm{L}$. is strongly influenced by several intrinsic and extrinsic factor including fertilizer, planting time, harvesting time and other agro-climatic factor (Singh and Misra 2000), (Singh et al 1995). To meet the increasing demand of $M$. spicata L. oil it urgently needs to increase its productivity. M. spicata L. has proved out to be of great commercial importance and consequently has attracted the attention of the researcher. It responds well to nitrogen (Tha and Singh 1979). Dan and Randhawa (2002) observed the application of nitrogen in conjugation of with phospho- 
rus resulted in increasing herbage yield and oil yield (Dan and Randhawa 2002). Types of harvesting time has great influenced on mentha yield and oil content of M. spicata L. Singh et al reported the importance of harvesting time of $M$. spicata $L$. on its herbage yield and oil content ( Singh et al 1991). The information regarding the effect of harvesting time and nitrogen-phosphorus nutrition of M. spicata L. is mearge in our local climatic condition. The present investigation, therefore was undertaken to ascertain the contribution of nitrogen-phosphorus fertilization and time of harvesting on the growth, yield and essential oil content and also physio-chemical characteristics of $M$. spicata L. oil.

\section{Materials and Methods}

The investigation was made in the field of BCSIR, Dhaka, during the period from March to November 2004. The eight nitrogen - phosphorus fertilizers were considered under study. The source of nitrogen - phosphorus were urea and triple super phosphate. The unit plot size was $2.1 \mathrm{~m} \times 2.1 \mathrm{~m}$. The row to row and plant to plant distance was $30 \mathrm{x}$ $30 \mathrm{~cm}$. The experiment was set in a randomized block design with three replications.
The soil properties of the experimental field are as below. Intercultural operation were done as per necessity. Data on plant height and number of branches per plant were recorded from 10 randomly selected plants in each plot and that on yield was recorded from the whole plot. For harvesting time 110 days $-T_{1}, 120$ days $-T_{2}, 130$ days $-T_{3}$, 140 days $-\mathrm{T}_{4}$ were used for the study. The nitrogen - phosphorus fertilizer doses under study were $60 \mathrm{~kg} \mathrm{~N} / \mathrm{ha}\left(\mathrm{N}_{1}\right), 80 \mathrm{~kg} \mathrm{~N} / \mathrm{ha}\left(\mathrm{N}_{2}\right)$,

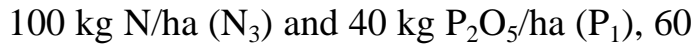
kg $\mathrm{P}_{2} \mathrm{O}_{5} /$ ha $\left(\mathrm{P}_{2}\right)$ and $80 \mathrm{~kg} \mathrm{P}_{2} \mathrm{O}_{5}$ /ha $\left(\mathrm{P}_{3}\right)$. The essential oil from the herb was extracted by the method of steam distillation. The freshly harvested herb from each plot was subjected to steam distillation for about three hours and the oil separated out form the distillate was dehydrated over anhydrous sodium sulphate where by a pale yellow volatile oil was obtained in pure form and each treatment was determined according to standard methods (Official Methods of Analysis of AOAC 1984). The collected data were statistically analyzed and the mean values were adjudged by Duncans New Multiple Range Test (Steel and Torrie1960).

\section{Physio-chemical properties of the soil in the experiment field}

\begin{tabular}{l|c|l|l|l|l|l|l|l|l|l|l}
\hline Texture & $\mathrm{pH}$ & $\begin{array}{l}\text { Organic } \\
\text { matter }\end{array}$ & $\begin{array}{l}\text { Total } \\
\text { nitrogen }\end{array}$ & \multicolumn{7}{|c}{ Available $\mu \mathrm{g} / \mathrm{g}$ soil } \\
\hline $\begin{array}{l}\text { Sandy } \\
\text { clay } \\
\text { loam }\end{array}$ & 7.2 & $1.52 \%$ & $0.04 \%$ & $\mathrm{~N}$ & $\mathrm{P}$ & $\mathrm{Na}$ & $\mathrm{Ca}$ & $\mathrm{Mg}$ & $\mathrm{Cu}$ & $\mathrm{Zn}$ & $\mathrm{K}$ \\
\cline { 4 - 10 } & & & & 79.3 & 0.45 & 20.3 & 135.2 & 242.9 & 4.08 & 7.5 & 21.3 \\
\hline
\end{tabular}




\section{Results and Discussion}

\section{Fertilizer effect}

The plant height is an important character which influence the herbage yield of $M$. spicata L. Plant height was maximum with $\mathrm{N}_{2} \mathrm{P}_{2}$ (Table I). The next value obtained from the plot received fertilizer at the rate of $80 \mathrm{~kg} \mathrm{~N}_{2}+40 \mathrm{~kg} \mathrm{P}_{2} \mathrm{O}_{5}\left(\mathrm{~N}_{2} \mathrm{P}_{1}\right)$. It is closely followed by $\mathrm{N}_{2} \mathrm{P}_{3}$ treatment. Minimum plant height per plant was obtained from control plants. Fertilizer applied at the rate of $80 \mathrm{~kg}$ $\mathrm{N}+60 \mathrm{~kg} \mathrm{P}_{2} \mathrm{O}_{5}\left(\mathrm{~N}_{2} \mathrm{P}_{2}\right)$ produced highest number of branches per plant. The next number of branch obtained from $\mathrm{N}_{2} \mathrm{P}_{3}$ treatment. The leaf length and leaf breath were highest at $\mathrm{N}_{2} \mathrm{P}_{2}$ and $\mathrm{N}_{2} \mathrm{P}_{3}$ treatment respectively. The value of leaf length and leaf breath were ranged from 3.6 to $4.3 \mathrm{~cm}$ and 2.4 to $3.3 \mathrm{~cm}$ respectively. The maximum herb yield was produced from the plot receiving fertilizer at the rate $80 \mathrm{~kg} \mathrm{~N} / \mathrm{ha}+60 \mathrm{~kg} \mathrm{P}_{2} \mathrm{O}_{5} / \mathrm{ha}$. This results were in close agreement with the Dan and Randhawa. The percentage of oil content M. spicata L. oil as affected by nitrogen phosphorus fertilizer treatment varied from $0.55 \%$ to $0.73 \%$. The highest percentage of oil obtained from $\mathrm{N}_{2} \mathrm{P}_{2}$ treatment. It is closely followed by $\mathrm{N}_{2} \mathrm{P}_{3}$ treatment. Additional doses of nitrogen phosphorus fertilizer showed decline response of mentha oil content. The moisture content of mentha oil

Table I. Effect of different level of nitrogen - phosphorus fertilization on the growth, herbage yield, oil content and physio - chemical characteristics of $M$. spicata L. oil

\begin{tabular}{|c|c|c|c|c|c|c|c|c|c|c|c|}
\hline $\begin{array}{c}\text { Fertiliz } \\
\text { er treat- } \\
\text { ment }\end{array}$ & $\begin{array}{c}\text { Plant } \\
\text { height } \\
(\mathrm{cm}) \\
\text { per } \\
\text { plant }\end{array}$ & $\begin{array}{c}\text { No. of } \\
\text { branch } \\
\text { per } \\
\text { plant }\end{array}$ & $\begin{array}{l}\text { Leaf } \\
\text { length } \\
(\mathrm{cm})\end{array}$ & $\begin{array}{c}\text { Leaf } \\
\text { breath } \\
(\mathrm{cm})\end{array}$ & $\begin{array}{l}\text { Total } \\
\text { fresh } \\
\text { herb } \\
(\mathrm{t} / \mathrm{ha})\end{array}$ & $\begin{array}{l}\text { Oil } \\
\text { (\%) }\end{array}$ & $\begin{array}{l}\text { Mois - } \\
\text { ture (\%) }\end{array}$ & $\begin{array}{l}\text { Acid } \\
\text { value }\end{array}$ & $\begin{array}{l}\text { Refrac- } \\
\text { tive } \\
\text { index at } \\
22^{\circ} \mathrm{C}\end{array}$ & $\begin{array}{l}\text { Density } \\
\text { at } 22^{\circ} \mathrm{C}\end{array}$ & $\begin{array}{c}\text { Optical } \\
\text { rota- } \\
\text { tion }\end{array}$ \\
\hline $\mathrm{N}_{0} \mathrm{P}_{0}$ & $53.1 c^{*}$ & 13.1c & $3.6 \mathrm{~b}$ & $2.4 \mathrm{~b}$ & $11.39 \mathrm{c}$ & $0.55 c$ & 73.70a & $2.75 a$ & $1.4732 \mathrm{a}$ & 0.9213a & -14.3a \\
\hline $\mathrm{N}_{1} \mathrm{P}_{1}$ & $57.1 \mathrm{~b}$ & $16.1 \mathrm{~b}$ & 3.9a & $2.6 \mathrm{~b}$ & $13.01 \mathrm{~b}$ & $0.61 \mathrm{c}$ & 73.76a & $2.76 a$ & $1.4736 \mathrm{a}$ & $0.9203 a$ & $-14.3 a$ \\
\hline $\mathrm{N}_{1} \mathrm{P}_{2}$ & $57.5 \mathrm{~b}$ & $18.2 \mathrm{~b}$ & 4.1a & 2.9ab & $13.21 \mathrm{~b}$ & $0.61 \mathrm{~b}$ & 69.10b & 2.76a & $1.4830 \mathrm{a}$ & $0.9206 a$ & $-14.4 a$ \\
\hline $\mathrm{N}_{1} \mathrm{P}_{3}$ & $58.5 b$ & $18.2 \mathrm{a}$ & $4.0 \mathrm{a}$ & 2.9ab & $13.20 \mathrm{a}$ & $0.61 \mathrm{~b}$ & $69.12 \mathrm{~b}$ & 2.75a & $1.4731 \mathrm{a}$ & $0.9204 a$ & $-14.1 \mathrm{a}$ \\
\hline $\mathrm{N}_{2} \mathrm{P}_{1}$ & 61.9a & 18.3a & $4.2 \mathrm{a}$ & 3.0a & 15.30a & $0.63 \mathrm{~b}$ & 73.71a & 2.76a & 1.4730a & $0.9214 \mathrm{a}$ & $-14.2 \mathrm{a}$ \\
\hline $\mathrm{N}_{2} \mathrm{P}_{2}$ & 62.6a & 19.8a & 4.3a & 3.1a & $15.39 a$ & 0.73a & 73.72a & 2.78a & $1.4828 \mathrm{a}$ & $0.9212 \mathrm{a}$ & $-14.3 a$ \\
\hline $\mathrm{N}_{2} \mathrm{P}_{3}$ & $61.8 \mathrm{a}$ & 19.6a & $4.2 \mathrm{a}$ & 3.3a & $15.41 \mathrm{a}$ & $0.72 \mathrm{a}$ & 69.01a & 2.78a & $1.4811 \mathrm{a}$ & $0.9212 \mathrm{a}$ & $-14.5 a$ \\
\hline $\mathrm{N}_{3} \mathrm{P}_{1}$ & $57.2 \mathrm{~b}$ & $16.2 \mathrm{~b}$ & $4.21 \mathrm{a}$ & $3.2 \mathrm{a}$ & 14.93ab & 0.71a & 73.04a & 2.78a & 1.4712a & 0.9211a & $-14.5 a$ \\
\hline $\mathrm{N}_{3} \mathrm{P}_{2}$ & $58.1 \mathrm{~b}$ & $16.1 \mathrm{~b}$ & 4.01a & 3.0a & $13.15 a b$ & $0.61 \mathrm{~b}$ & $69.20 \mathrm{~b}$ & 2.76a & $1.4720 \mathrm{a}$ & $0.9212 \mathrm{a}$ & $-14.3 a$ \\
\hline $\mathrm{N}_{3} \mathrm{P}_{3}$ & $58.1 \mathrm{~b}$ & $16.0 \mathrm{~b}$ & 4.1a & 3.1a & $13.17 \mathrm{~b}$ & $0.60 \mathrm{~b}$ & $69.21 \mathrm{~b}$ & 2.70a & 1.4711a & 0.9211a & $-14.4 a$ \\
\hline
\end{tabular}

* Means with the same letter are not different from one another at the $5 \%$ level of significance. 
ranged from 69.01 to $73.76 . \mathrm{N}_{1} \mathrm{P}_{1}$ treatment produced highest value (73.76) of moisture content. The refractive index value ranged from 1.4711 to 1.4830 . The highest refractive index obtained from $\mathrm{N}_{1} \mathrm{P}_{2}$ treatment. It is closely followed by $\mathrm{N}_{2} \mathrm{P}_{2}$ treatment. The optical rotation and density of oil obtained from each treatment did not show any significant change with different fertilizer treatment. Shahidullah et al (1997) reported similar result regarding optical rotation and density of oil of $M$. spicata L.

\section{Effect of harvest time :}

The results on the effect of harvest time on growth, yield component, herb yield and oil content of M. spicata L. have been presented in Table II. Results indicate that plant height increased progressively with increase in age of plants and the maximum plant height $(64.1 \mathrm{~cm})$ attended at the age of 140 days of the herbs. It was significantly higher from the rest of the harvesting time. The next higher value came from 130 days. The number of branches per plant did not differ significantly due to change of stage of harvesting. The highest leaf length was $4.2 \mathrm{~cm}$ and leaf breath was $3.2 \mathrm{~cm}$ at 130 day harvest. The earlier harvest showed lower values of leaf length and breath. Herbage yield increased progressively with the increase in age of plant up to 130 days. The highest yield was noticed in 130 days. The results are in agreement with those of Raj et al (2003). The oil content of M. spicata L. increased progressively with increaseing of the harvest time up to 130 days after which the value declined. This next value obtained from 120 days harvest time. Raj et al (2003) found higher oil content in mentha at 130 days of harvesting. The youngest herbs gave the lower value of oil content. The moisture is maximum at 120 days of harvest. It was closely followed by 130 days of harvest. The

Table II. Effect of harvesting time on the growth, herbage yield, oil content and physio chemical characteristics of $M$. spicata $\mathbf{L}$. oil.

\begin{tabular}{c|c|c|c|c|c|c|c|c|c|c|c}
\hline $\begin{array}{c}\text { Harves- } \\
\text { ting } \\
\text { time } \\
\text { (days) }\end{array}$ & $\begin{array}{c}\text { Plant } \\
\text { height } \\
(\mathrm{cm}) \\
\text { per } \\
\text { plant }\end{array}$ & $\begin{array}{c}\text { No. of } \\
\text { branch } \\
\text { per } \\
\text { plant }\end{array}$ & $\begin{array}{c}\text { Leaf } \\
\text { length } \\
(\mathrm{cm})\end{array}$ & $\begin{array}{c}\text { Leaf } \\
\text { breath } \\
(\mathrm{cm})\end{array}$ & $\begin{array}{c}\text { Total } \\
\text { fresh } \\
\text { herb } \\
(\mathrm{t} / \mathrm{ha})\end{array}$ & $\begin{array}{c}\text { Oil } \\
(\%)\end{array}$ & $\begin{array}{c}\text { Mois }- \\
\text { ture }(\%)\end{array}$ & $\begin{array}{c}\text { Acid } \\
\text { value }\end{array}$ & $\begin{array}{c}\text { Refrac- } \\
\text { tive } \\
\text { index at } \\
22^{\circ} \mathrm{c}\end{array}$ & $\begin{array}{c}\text { Density } \\
\text { at 22 }\end{array}$ & $\begin{array}{c}\text { Optical } \\
\text { rota- } \\
\text { tion }\end{array}$ \\
\hline 110 & $60.2 \mathrm{c}^{*}$ & $18.3 \mathrm{a}$ & $4.0 \mathrm{a}$ & $2.9 \mathrm{a}$ & $14.01 \mathrm{~b}$ & $0.63 \mathrm{a}$ & $73.60 \mathrm{a}$ & $2.75 \mathrm{a}$ & $1.4670 \mathrm{a}$ & $0.9201 \mathrm{a}$ & $-14.2 \mathrm{a}$ \\
120 & $62.0 \mathrm{~b}$ & $18.6 \mathrm{a}$ & $4.0 \mathrm{a}$ & $3.0 \mathrm{a}$ & $14.30 \mathrm{ab}$ & $0.63 \mathrm{a}$ & $73.75 \mathrm{a}$ & $2.76 \mathrm{a}$ & $1.4690 \mathrm{a}$ & $0.9201 \mathrm{a}$ & $-14.3 \mathrm{a}$ \\
130 & $62.2 \mathrm{~b}$ & $18.5 \mathrm{a}$ & $4.2 \mathrm{a}$ & $3.2 \mathrm{a}$ & $15.30 \mathrm{a}$ & $0.72 \mathrm{a}$ & $73.70 \mathrm{a}$ & $2.75 \mathrm{a}$ & $1.4730 \mathrm{a}$ & $0.9202 \mathrm{a}$ & $-14.2 \mathrm{a}$ \\
140 & $64.1 \mathrm{a}$ & $18.6 \mathrm{a}$ & $4.1 \mathrm{a}$ & $3.1 \mathrm{a}$ & $15.10 \mathrm{a}$ & $0.70 \mathrm{a}$ & $72.62 \mathrm{a}$ & $2.75 \mathrm{c}$ & $1.4821 \mathrm{a}$ & $0.9203 \mathrm{a}$ & $-14.4 \mathrm{a}$ \\
\hline
\end{tabular}

* Means with the same letter are not different from one another at the $5 \%$ level of significance. 
refractive index was also higher when harvesting was done after 140 days. Refractive index of oil was higher by increasing harvesting time. Raj et al (2003) recorded the similar results of refractive index.

\section{Interaction effect of fertilizer and harvest time}

In case of interaction effect the highest plant height was obtained from the plot receiving the fertilizer at the rate of $80 \mathrm{~kg} \mathrm{~N}+60 \mathrm{~kg}$ $\mathrm{P}_{2} \mathrm{O}_{5}\left(\mathrm{~N}_{2} \mathrm{P}_{2}\right)$ per hectare with 130 days harvesting (Table III). It is closely followed by $\mathrm{N}_{2} \mathrm{P}_{3}$ treatment with the same harvesting period. Fertilizer applied at the rate of $100 \mathrm{~kg}$ $\mathrm{N}+80 \mathrm{~kg} \mathrm{P}_{2} \mathrm{O}_{5}\left(\mathrm{~N}_{3} \mathrm{P}_{3}\right)$ per hectare with 130 days harvesting time produced highest number of branches. The maximum herbage yield obtained from the plot which received the fertilizer at the rate of $100 \mathrm{~kg} \mathrm{~N}+60 \mathrm{~kg}$
$\mathrm{P}_{2} \mathrm{O}_{5}\left(\mathrm{~N}_{3} \mathrm{P}_{2}\right)$ per hectare within the harvestperiod of 130 days. The results are in partial agreement with those reported by K. Singh et al who recorded the maximum herbage yield of $M$. spicata L. by applying nitrogen at the rate of $100 \mathrm{~kg} \mathrm{~N}$ per hectare within the harvest period of 130 days. Oil content of $M$. spicata L. as affected by N-P fertilizer treatment and harvest time varied from $0.55 \%$ $0.79 \%$. The maximum amount of oil of $M$. spicata L. obtained from $\mathrm{N}_{2} \mathrm{P}_{2}$ treatment at 130 days harvest. The next higher value of mentha oil noted from $\mathrm{N}_{2} \mathrm{P}_{1}$ treatment within same harvest period. The percentage of moisture ranges from 72.02-75.65. The higher harvesting time enhanced the refractive index. The acid value, optical rotation, density of oil obtained from each treatment did not show any significant change with different fertilizer treatment and harvesting time.

Table III. Interaction effect of nitrogen-phosphorus fertilization and time of harvest on growth, herbage yield, oil content and physio-chemical characteristics of $M$. spicata L. oil

\begin{tabular}{|c|c|c|c|c|c|c|c|c|c|c|}
\hline $\begin{array}{c}\text { Harves } \\
\text { ting } \\
\text { time } \\
\text { (days) }\end{array}$ & \begin{tabular}{|c|} 
Fertiliz \\
er treat- \\
ment
\end{tabular} & $\begin{array}{l}\text { Plant } \\
\text { height } \\
(\mathrm{cm})\end{array}$ & $\begin{array}{l}\text { No. of } \\
\text { branch } \\
\text { per } \\
\text { plant }\end{array}$ & $\begin{array}{l}\text { Total } \\
\text { fresh } \\
\text { herb } \\
(\mathrm{t} / \mathrm{ha})\end{array}$ & $\begin{array}{l}\text { Oil } \\
(\%)\end{array}$ & $\begin{array}{c}\text { Mois- } \\
\text { ture } \\
(\%)\end{array}$ & $\begin{array}{l}\text { Acid } \\
\text { value }\end{array}$ & $\begin{array}{c}\text { Refrac- } \\
\text { tive } \\
\text { index at } \\
22^{\mathrm{O}} \mathrm{C}\end{array}$ & $\begin{array}{l}\text { Density } \\
\text { at } 22^{\circ} \mathrm{C}\end{array}$ & $\begin{array}{l}\text { Optical } \\
\text { rotation }\end{array}$ \\
\hline \multirow{10}{*}{110} & $\mathrm{NoP}_{0}$ & $53.4 d^{*}$ & $13.2 \mathrm{c}$ & $11.30 \mathrm{c}$ & $0.55 \mathrm{~d}$ & $72.18 \mathrm{~b}$ & 2.71a & 1.4601a & $0.9203 a$ & $-14.30 a$ \\
\hline & $\mathrm{N}_{1} \mathrm{P}_{1}$ & $58.2 \mathrm{c}$ & $16.2 \mathrm{~b}$ & $13.10 \mathrm{bc}$ & $0.60 c$ & $72.18 \mathrm{~b}$ & $2.75 a$ & $1.4670 \mathrm{a}$ & $0.9203 a$ & $-14.31 \mathrm{a}$ \\
\hline & $\mathrm{N}_{1} \mathrm{P}_{2}$ & $58.3 \mathrm{c}$ & $16.2 \mathrm{~b}$ & $13.40 \mathrm{bc}$ & $0.61 \mathrm{c}$ & $72.17 \mathrm{~b}$ & $2.75 a$ & $1.4610 \mathrm{a}$ & 0.9231a & $-14.41 a$ \\
\hline & $\mathrm{N}_{1} \mathrm{P}_{3}$ & $59.2 \mathrm{c}$ & $16.3 \mathrm{~b}$ & $13.25 \mathrm{bc}$ & $0.60 c$ & $73.10 \mathrm{~b}$ & 2.76a & $1.4611 \mathrm{a}$ & $0.9201 \mathrm{a}$ & $-14.19 a$ \\
\hline & $\mathrm{N}_{2} \mathrm{P} 1$ & $59.0 \mathrm{c}$ & $16.3 \mathrm{~b}$ & $13.15 b c$ & $0.60 \mathrm{c}$ & $73.10 \mathrm{~b}$ & $2.75 a$ & $1.4610 \mathrm{a}$ & $0.9201 \mathrm{a}$ & $-14.21 \mathrm{a}$ \\
\hline & $\mathrm{N}_{2} \mathrm{P}_{2}$ & $60.1 \mathrm{c}$ & $16.4 \mathrm{~b}$ & $13.17 \mathrm{bc}$ & $0.60 c$ & $72.10 \mathrm{~b}$ & 2.76a & $1.4612 \mathrm{a}$ & $0.9231 \mathrm{a}$ & $-14.50 \mathrm{a}$ \\
\hline & $\mathrm{N}_{2} \mathrm{P}_{3}$ & $60.1 \mathrm{c}$ & 17.1ab & $12.27 \mathrm{bc}$ & $0.61 \mathrm{c}$ & $72.20 \mathrm{~b}$ & $2.77 a$ & $1.4610 \mathrm{a}$ & $0.9221 \mathrm{a}$ & $-14.42 a$ \\
\hline & $\mathrm{N}_{3} \mathrm{P} 1$ & $60.1 \mathrm{c}$ & 17.1ab & $14.40 \mathrm{~b}$ & $0.66 \mathrm{~b}$ & $72.15 \mathrm{~b}$ & $2.79 a$ & $1.4615 \mathrm{a}$ & $0.9212 \mathrm{a}$ & $-14.31 a$ \\
\hline & $\mathrm{N}_{3} \mathrm{P}_{2}$ & $60.2 \mathrm{c}$ & 17.2ab & $14.60 \mathrm{~b}$ & $0.67 \mathrm{~b}$ & $72.13 \mathrm{~b}$ & $2.75 a$ & $1.4617 \mathrm{a}$ & $0.9224 a$ & $-14.60 \mathrm{a}$ \\
\hline & $\mathrm{N}_{3} \mathrm{P}_{3}$ & $60.2 \mathrm{c}$ & 17.1ab & $13.15 b c$ & $0.65 b$ & $72.14 \mathrm{~b}$ & $2.76 \mathrm{a}$ & $1.4616 \mathrm{a}$ & 0.9213a & $-14.20 \mathrm{a}$ \\
\hline
\end{tabular}


Table III to be contd.

\begin{tabular}{|c|c|c|c|c|c|c|c|c|c|c|}
\hline \multirow{9}{*}{120} & $\mathrm{~N}_{1} \mathrm{P}_{1}$ & 64.2a & $18.4 \mathrm{a}$ & $13.24 \mathrm{bc}$ & $0.66 \mathrm{~b}$ & $74.95 a$ & $2.73 a$ & $1.4651 \mathrm{a}$ & $0.9201 \mathrm{a}$ & $-14.2 \mathrm{a}$ \\
\hline & $\mathrm{N}_{1} \mathrm{P}_{2}$ & 63.1a & $18.2 \mathrm{a}$ & $14.61 b$ & $0.67 \mathrm{~b}$ & $72.14 \mathrm{~b}$ & 2.76a & $1.4650 \mathrm{a}$ & $0.9205 a$ & $-14.5 a$ \\
\hline & $\mathrm{N}_{1} \mathrm{P}_{3}$ & 60.1ab & 18.3a & $14.50 \mathrm{~b}$ & $0.65 \mathrm{~b}$ & 74.81a & $2.75 a$ & $1.4652 \mathrm{a}$ & $0.9203 a$ & $-14.2 \mathrm{a}$ \\
\hline & $\mathrm{N}_{2} \mathrm{P}_{1}$ & 62.2ab & 18.1a & $14.40 \mathrm{~b}$ & $0.66 \mathrm{~b}$ & 75.11a & 2.76a & $1.4655 \mathrm{a}$ & $0.9234 a$ & $-14.1 \mathrm{a}$ \\
\hline & $\mathrm{N}_{2} \mathrm{P}_{2}$ & $60.2 \mathrm{ab}$ & 18.2a & $14.50 \mathrm{~b}$ & $0.67 \mathrm{~b}$ & $72.10 \mathrm{~b}$ & $2.75 a$ & $1.4652 \mathrm{a}$ & $0.9204 a$ & $-14.2 \mathrm{a}$ \\
\hline & $\mathrm{N}_{2} \mathrm{P}_{3}$ & $60.1 \mathrm{c}$ & $18.4 \mathrm{a}$ & $14.20 \mathrm{~b}$ & $0.66 \mathrm{~b}$ & 75.11a & $2.75 a$ & $1.4653 \mathrm{a}$ & $0.9236 a$ & $-14.4 \mathrm{a}$ \\
\hline & $\mathrm{N}_{3} \mathrm{P}_{1}$ & $60.1 \mathrm{c}$ & 18.3a & $16.25 a$ & $0.67 \mathrm{~b}$ & 75.15a & $2.77 a$ & $1.4652 \mathrm{a}$ & $0.9217 a$ & $-14.3 a$ \\
\hline & $\mathrm{N}_{3} \mathrm{P}_{2}$ & 62.3ab & 18.2a & 16.11a & $0.69 \mathrm{~b}$ & 72.16b & 2.76a & $1.4654 \mathrm{a}$ & $0.9226 a$ & $-14.4 \mathrm{a}$ \\
\hline & $\mathrm{N}_{3} \mathrm{P}_{3}$ & $61.9 \mathrm{~b}$ & 18.1a & $14.20 \mathrm{~b}$ & 0.70a & $75.41 \mathrm{a}$ & $2.77 \mathrm{a}$ & $1.4653 \mathrm{a}$ & $0.9237 \mathrm{a}$ & $-14.3 \mathrm{a}$ \\
\hline \multirow{9}{*}{130} & $\mathrm{~N}_{1} \mathrm{P}_{1}$ & 62.1ab & 18.3a & $14.30 \mathrm{~b}$ & $0.77 \mathrm{a}$ & $72.14 \mathrm{~b}$ & $2.75 a$ & $1.4702 \mathrm{a}$ & $0.9205 a$ & $-14.2 \mathrm{a}$ \\
\hline & $\mathrm{N}_{1} \mathrm{P}_{2}$ & 62.0ab & 18.1a & $14.41 \mathrm{~b}$ & $0.76 a$ & $75.65 a$ & $2.75 a$ & $1.4710 \mathrm{a}$ & $0.9235 a$ & $-14.5 a$ \\
\hline & $\mathrm{N}_{1} \mathrm{P}_{3}$ & 62.1ab & 18.4a & $16.40 \mathrm{a}$ & $0.77 a$ & 75.64a & $2.75 a$ & $1.4711 \mathrm{a}$ & $0.9215 a$ & $-14.2 \mathrm{a}$ \\
\hline & $\mathrm{N}_{2} \mathrm{P}_{1}$ & 62.2ab & 18.2a & $15.90 \mathrm{ab}$ & 0.78a & $74.15 \mathrm{a}$ & 2.70a & $1.4713 \mathrm{a}$ & $0.9214 a$ & $-14.3 a$ \\
\hline & $\mathrm{N}_{2} \mathrm{P}_{2}$ & 64.3a & 19.8a & $15.91 \mathrm{ab}$ & $0.79 a$ & $74.10 \mathrm{a}$ & $2.75 a$ & $1.4714 \mathrm{a}$ & 0.9213a & $-14.4 \mathrm{a}$ \\
\hline & $\mathrm{N}_{2} \mathrm{P}_{3}$ & $64.2 \mathrm{a}$ & $19.7 \mathrm{a}$ & 16.51a & 0.76a & 74.16a & 2.72a & 1.4713a & $0.9211 \mathrm{a}$ & $-14.0 \mathrm{a}$ \\
\hline & $\mathrm{N}_{3} \mathrm{P}_{1}$ & 64.1a & $19.7 \mathrm{a}$ & $14.12 \mathrm{~b}$ & $0.76 \mathrm{a}$ & $72.02 b$ & 2.73a & $1.4714 \mathrm{a}$ & $0.9225 a$ & $-14.2 \mathrm{a}$ \\
\hline & $\mathrm{N}_{3} \mathrm{P}_{2}$ & 63.9a & 19.9a & $14.40 \mathrm{~b}$ & $0.77 a$ & 72.03b & 2.72a & $1.4713 a$ & $0.9225 a$ & $-14.1 \mathrm{a}$ \\
\hline & $\mathrm{N}_{3} \mathrm{P}_{3}$ & 63.2a & 19.8a & $13.39 \mathrm{bc}$ & 0.76a & 74.16a & 2.73a & 1.4713a & $0.9227 a$ & $-14.4 \mathrm{a}$ \\
\hline \multirow{9}{*}{140} & $\mathrm{~N}_{1} \mathrm{P}_{1}$ & $60.1 \mathrm{c}$ & 17.2ab & $14.41 \mathrm{~b}$ & $0.67 \mathrm{~b}$ & 75.43a & $2.71 \mathrm{a}$ & 1.4810a & $0.9221 \mathrm{a}$ & $-14.4 a$ \\
\hline & $\mathrm{N}_{1} \mathrm{P}_{2}$ & 62.1ab & 17.2ab & $14.24 \mathrm{~b}$ & $0.66 \mathrm{~b}$ & 72.14b & $2.77 \mathrm{a}$ & $1.4809 \mathrm{a}$ & $0.9231 \mathrm{a}$ & $-14.3 \mathrm{a}$ \\
\hline & $\mathrm{N}_{1} \mathrm{P}_{3}$ & 62.2ab & 17.1ab & $14.30 \mathrm{~b}$ & $0.68 \mathrm{~b}$ & 72.13b & $2.78 \mathrm{a}$ & $1.4810 \mathrm{a}$ & $0.9213 a$ & $-14.6 \mathrm{a}$ \\
\hline & $\mathrm{N}_{2} \mathrm{P}_{1}$ & 62.1ab & 18.1ab & $14.40 \mathrm{~b}$ & $0.67 \mathrm{~b}$ & 72.14b & 2.79a & $1.4815 \mathrm{a}$ & $0.9214 \mathrm{a}$ & $-14.2 \mathrm{a}$ \\
\hline & $\mathrm{N}_{2} \mathrm{P}_{2}$ & 61.1ab & 17.2ab & $13.12 \mathrm{bc}$ & $0.68 \mathrm{~b}$ & 75.11a & $2.75 a$ & $1.4814 \mathrm{a}$ & $0.9221 \mathrm{a}$ & $-14.3 \mathrm{a}$ \\
\hline & $\mathrm{N}_{2} \mathrm{P}_{3}$ & 60.1c & 17.3ab & $13.12 \mathrm{bc}$ & $0.68 \mathrm{~b}$ & $72.11 \mathrm{~b}$ & 2.71a & $1.4812 \mathrm{a}$ & $0.9220 \mathrm{a}$ & $-14.2 \mathrm{a}$ \\
\hline & $\mathrm{N}_{3} \mathrm{P}_{1}$ & $60.1 \mathrm{c}$ & 17.1ab & $14.50 \mathrm{~b}$ & $0.67 \mathrm{~b}$ & 72.16b & 2.75a & $1.4814 \mathrm{a}$ & 0.9213a & $-14.1 \mathrm{a}$ \\
\hline & $\mathrm{N}_{3} \mathrm{P}_{2}$ & 62.1ab & 17.2ab & $14.10 \mathrm{~b}$ & $0.67 b$ & 75.15a & 2.76a & 1.4813a & 0.9210a & $-14.4 a$ \\
\hline & $\mathrm{N}_{3} \mathrm{P}_{3}$ & 62.2ab & 18.1a & $14.30 \mathrm{~b}$ & $0.67 b$ & 72.14b & $2.76 a$ & $1.4814 \mathrm{a}$ & 0.9213a & $-14.2 \mathrm{a}$ \\
\hline
\end{tabular}

* Means with the same letter are not different from one another at the $5 \%$ level of significance. 


\section{Conclusion}

From the present study it can be concluded that $M$. spicata L. has presently grown an experimental basis to evaluate its potential for commercial cultivation in our country. The results of the study suggested that growth and yield of $M$. spicata $\mathrm{L}$. herbs as well as oil yield could significantly be improved by the application of NP fertilizer and proper harvesting time (130 days). Agronomic treatmemts for mentha species had not been standardized in our country. So further researches are needed in this direction with different mentha species to establish the present findings.

\section{Acknowledgement}

The authors are grateful to Director, BCSIR Laboratories, Dhaka for his interest during the progress of the work. They do acknowledge assistance undered by Abul Kasem for collection and preparation of the plant samples.

\section{Reference}

Chattpadhyay, A. Prasad, A. and Gupta, N (2002). Response of spearment (Mentha spicata L) to sulpher fertilization. J. of Medicinal and Aromatic Plant Sciences 22:450-452.

Dan, V.K. and Randhawa, G.S. (2002). Nitrogen requirement of different mentha species in potato, mentha species. I. effect on growth and yield. Indian Perfume 46 (3) 251-57.

Official Methods of Analysis of AOAC (1984). 14th edition.

Raj, V. Vikrant, Sharma, A. Singh B.P. (2003). Effect of nitrogen levels and harvest times on oil content and oil yield of Japanese mint. Annals of Agri. Bio. Research 8 (2): 185-187.

Raj, V. Vikrant, Singh, B.P. (2003). Physiocochemical properties of oil and nitrogen concentration of Japanese mint as affected by nitrogen and harvesting time. Annals of Agri. Bio. Research 8 (2): 189-192.

Steel, C.G.D. and Torrie, J.H. (1960 ). Principles and Procedures of Statistics. Mc. Graw Hill Book Co. Inc. New York. 109.

Shahidullah, M. Haq, M.F. Karim, M.A. and Nada, M. K. (1997). Response of Mentha spicata to different levels of nitrogen. B.J.Sci.Ind.Res. 32 (2): 292-294.

Sing, P. and Misra, A. (2000). Influence of graded level of iron on growth and essential oil production in Mentha spicata L. J. of Medicinal and Aromatic Plant Science 22: 557-562.

Singh, M. Singh, V.P. Singh, D.V. (1995). Effect of planting time on growth, yield and quality of spearmint (Mentha spicata L.) under sub-tropical climate of central Uttar 
Pradesh. J. of Essential Oil Research. 7 (6): 621-626.

Singh, K. Kothari, S.K. Chauchan, H.S. and Singh, J.P. (1991). Effect of harvesting date and nitrogen on growth, light utilization, nitrogen use efficiency and oil yield of spearmint. Int.J .Trop. Agric. 10: 282-291.
Tha, P.K. and Singh, N.P. (1979). Response of Mentha spicata L. ( Spearment) to different levels of nitrogen application and row spacing. Indian Perfume. 23: 95-99.

Received : May 03, 2007;

Accepted : November 20, 2007 